\title{
Doenças crônicas não transmissíveis e fatores associados em adultos numa área urbana de pobreza do nordeste brasileiro
}

\author{
Chronic noncommunicable diseases and associated factors among \\ adults in an impoverished urban area of the Brazilian northeast
}

\author{
Silvia Pereira da Silva de Carvalho Melo (https://orcid.org/0000-0002-8162-1743) ${ }^{1}$ \\ Eduarda Ângela Pessoa Cesse (http://orcid.org/0000-0001-5745-3981) ${ }^{1}$ \\ Pedro Israel Cabral Lira (https://orcid.org/0000-0002-1534-1620) ${ }^{2}$ \\ Anete Rissin (https://orcid.org/0000-0002-2135-177X) ${ }^{3}$ \\ Rachel de Sá Barreto Luna Callou Cruz (https://orcid.org/0000-0002-4596-313X) ${ }^{3}$ \\ Malaquias Batista Filho (https://orcid.org/0000-0002-1490-0590) ${ }^{3}$
}

${ }^{1}$ Instituto Aggeu Magalhães, Fiocruz. Av. Moraes Rego s/n Pavilhão Josué de Castro, Cidade Universitária. 50670420 Recife PE Brasil. silviaps2008@gmail.com ${ }^{2}$ Centro de Ciências da Saúde, Universidade Federal de Pernambuco. Recife PE Brasil.

${ }^{3}$ Diretoria de Pesquisas, Instituto de Medicina Integral Prof. Fernando Figueira. Recife PE Brasil.

\begin{abstract}
The scope of this study is to analyze the prevalence and factors associated with Chronic Noncommunicable Diseases (CNCD) in adults living in an impoverished urban area located in Recife in the Brazilian northeast. It is a cross-sectional study with a sample of 631 adults of 20 to 59 years of age. The possible associations of CNCD with demographic, socioeconomic, behavioral and health-related factors were analyzed using Poisson Regression, considering a p value of $<0.05$ as being statistically significant. The prevalence of CNCD was 56.7\%; highest among males (60.8\%); adults aged 50-59 years (80.5\%); lower economic class (57.7\%); and lower level of schooling (62\%). The problem was also associated with individuals with $B M I \geq 25 \mathrm{~kg} / \mathrm{m} 2$ (34.2\%) as well as individuals who reported poor health status (76.4\%). In the multivariate hierarchical model, the statistically significant variables were: schooling, BMI, health perception, gender and age bracket. A high prevalence of at least one CNCD was observed, as well as a statistically significant association between CNCD and the following variables: schooling, BMI, health perception, gender and age bracket. These results suggest the need to intensify health promotion actions in poor communities, aiming at enhanced control of health in general.
\end{abstract}

Key words Chronic disease, Risk factors, Poverty
Resumo Objetiva-se analisar a prevalência e os fatores associados às Doenças Crônicas não Transmissíveis (DCNT), em adultos residentes numa área urbana de pobreza situada em Recife, Nordeste do Brasil. Trata-se de um estudo transversal, com amostra de 631 adultos de 20 a 59 anos. Analisaram-se possíveis associações das DCNT com fatores demográficos, socioeconômicos, comportamentais e relativos à saúde, por meio de Regressão de Poisson, considerando-se como estatisticamente significantes aqueles com valor de $p<0,05$. A prevalência de DCNT foi de 56,7\%, sendo maior no sexo masculino (60,8\%), entre os adultos com 50-59 anos (80,5\%), de menor classe econômica (57,7\%) e menor nível de instrução (62\%). O problema também predominou entre aqueles com $I M C \geq 25 \mathrm{Kg} / \mathrm{m}^{2}(34,2 \%)$ e que referiram estado de saúde ruim $(76,4 \%)$. No modelo multivariado hierarquizado, as variáveis estatisticamente significantes foram: escolaridade, IMC, percepção da própria saúde, sexo e faixa etária. Observouse, neste estudo, uma elevada prevalência de pelo menos uma DCNT, bem como, associação estatisticamente significante entre DCNT e as variáveis: escolaridade, IMC, percepção da própria saúde, sexo e faixa etária. Estes resultados sugerem a necessidade de se intensificar as ações de promoção à saúde, em comunidades carentes, com vistas ao seu melhor controle.

Palavras-chave Doença crônica, Fatores de risco, Pobreza 
A situação de saúde no Brasil se caracteriza por uma transição demográfica acelerada e por um perfil epidemiológico de tripla carga de doenças (uma agenda não superada de doenças infecciosas e carenciais, uma carga importante de causas externas e uma marcante presença das condições crônicas), trazendo neste contexto as Doenças Crônicas Não Transmissíveis (DCNT) ${ }^{1}$. Fazem parte do grupo das DCNT as doenças cerebrovasculares, cardiovasculares, dislipidemias, diabetes mellitus, doenças respiratórias obstrutivas e neoplasias, responsáveis pelas maiores taxas de mortalidade no mundo ${ }^{2-4}$.

Essas doenças atingem indivíduos de todas as camadas socioeconômicas e, de forma mais intensa, aqueles pertencentes a grupos vulneráveis, como os de baixa escolaridade e renda ${ }^{5,6}$. Conforme a Organização Mundial de Saúde (OMS), essas doenças criam um círculo vicioso com a pobreza, impactando negativamente sobre o desenvolvimento macroeconômico dos países, especialmente daqueles de média e baixa renda ${ }^{7}$.

Em 2008, as DCNT foram responsáveis por $63 \%$ dos óbitos no mundo, constituindo-se na primeira causa de mortalidade, com predominância de mortalidade prematura, principalmente em populações mais pobres ${ }^{8,9}$. Já no Brasil, a estimativa foi de $79,8 \%$ das mortes, no ano de $2014^{10}$.

Em estudo sobre a carga global das doenças, no Brasil, realizado por Leite et al. ${ }^{11}$, foi demonstrado que $77,2 \%$ dos anos de vida perdidos por incapacidade eram representados pelo grupo das DCNT. As piores situações foram encontradas nas regiões Norte e Nordeste, o que pode ser reflexo de piores condições de vida e de acesso aos serviços de saúde, dificultando o controle dos fatores de risco para o tratamento das doenças, determinando maior incidência de sequelas e mortalidade precoce.

As DCNT compartilham diversos fatores de risco, como hereditariedade, raça, sexo, tabagismo, consumo excessivo de álcool, dislipidemias, consumo insuficiente de frutas, legumes e verduras e sedentarismo ${ }^{12}$. A inatividade física e o excesso de peso são responsáveis, respectivamente, por 3,2 e 2,8 milhões de mortes/ano. Ao tabagismo e ao consumo abusivo de álcool são atribuídas 2,3 e 6 milhões de mortes ao $\mathrm{ano}^{13}$. O monitoramento das DCNT e de seus fatores de risco é prioridade no Brasil, e acompanha os esforços globais que estão sendo desenvolvidos ${ }^{8}$. A vigilância epidemiológica dessas doenças é essencial, uma vez que propicia o melhor entendimento, de distribuição, magnitude e tendência desses fatores ${ }^{14}$; contudo, essa ação é ainda incipiente em áreas urbanas de pobreza (fa- velas). No Brasil, cerca de 11 milhões de pessoas moram nessas áreas (6\%), segundo o Instituto Brasileiro de Geografia e Estatística - IBGE ${ }^{15}$.

De acordo com uma recente publicação da revista "Lancet", o crescimento maciço das favelas no mundo não foi acompanhado por um aumento proporcional de estudos científicos, que permanece rudimentar quando comparado com outras realizadas em áreas não faveladas ${ }^{16}$. Reconhecendo-se a escassez de estudos nessas áreas $\mathrm{e}$ o seu impacto no processo saúde/doença da população, justifica-se o presente estudo, que objetivou analisar a prevalência e os fatores associados às DCNT, em adultos com idade de 20 a 59 anos, residentes numa área urbana de pobreza, situada em Recife, Nordeste do Brasil.

\section{Métodos}

Foi realizado um estudo de corte transversal utilizando dados da pesquisa "Saúde, nutrição e serviços assistenciais numa população favelada do Recife: um estudo baseline", desenvolvido pelo Instituto de Medicina Integral Professor Fernando Figueira - IMIP, em parceria com o Departamento de Nutrição da Universidade Federal de Pernambuco - UFPE e Prefeitura do Recife. É um inquérito proposto para estabelecer a linha de base de um projeto de coorte com intervenção para os próximos dez anos, especificamente focado nos problemas de saúde de interesse dominante (ou prioritários) na época investigada. A coleta de dados foi de base domiciliar e ocorreu entre junho a dezembro de 2014, numa área conhecida como Comunidade dos Coelhos, município do Recife, capital do Estado de Pernambuco. Para o cálculo da amostra tomou-se como referência um universo de 3.816 adultos com idade entre 20 e 59 anos, estimado pelo Sistema de Informação de Atenção Básica (SIAB) - Recife. Foi estimada uma prevalência de $50 \%$, um erro amostral de $4 \%$ e um nível de confiança de $95 \%$. Assim obteve-se uma amostra 498 adultos para a qual foi acrescentado um percentual de $20 \%$ para compensar possíveis perdas e/ou inconsistências dos questionários, resultando assim, numa amostra de 623 indivíduos. A amostra final, entretanto, contou com um total de 631 adultos. A amostragem foi do tipo probabilística e os adultos foram indicados por sorteio aleatório simples, a partir de uma listagem numerada dos adultos de 20 a 59 anos residentes dos domicílios, numa relação de um em cada quatro pessoas, sem substituição. $\mathrm{Na}$ ausência do indivíduo indicado no sorteio, eram realizadas mais duas visitas; se ainda assim 
o indivíduo não fosse encontrado no domicílio não era permitida a substituição. Foram excluídas as gestantes, os indivíduos com limitação física congênita ou adquirida que impossibilitassem a aferição das medidas antropométricas e os casos com edema visível ou transtornos psíquicos que dificultassem a colaboração.

Uma vez que o presente estudo procurou identificar as variáveis associadas com as DCNT, cálculos a posteriori foram realizados para estimar as diferenças do conjunto de variáveis independentes. Para tanto, considerou-se um erro de 1de $95 \%$ e poder de estudo 1- de $80 \%$. Assim, este estudo teria um poder suficiente para detectar razões de prevalência de 1,33 ou mais como fator de associação. Para o cálculo da amostra foi utilizado o programa estatístico Epi info versão 6.04 para estudos transversais.

A variável dependente foi construída pela presença de pelo menos uma das seguintes DCNT: Diabetes Mellitus (DM), Hipertensão Arterial Sistêmica (HAS), dislipidemia. Para o DM e dislipidemia foram realizados exames bioquímicos (glicemia de jejum, LDL-colesterol e triglicerídeos). Em relação ao DM, os adultos com glicemia sanguínea maior ou igual a $126 \mathrm{mg} / \mathrm{dL}$ ou com relato de uso prescrito de hipoglicemiante foram classificados como diabéticos ${ }^{17}$. Aqueles como valor do LDL-colesterol $\geq 160 \mathrm{mg} / \mathrm{dL}$ ou valor dos triglicerídeos $\geq 150 \mathrm{mg} / \mathrm{dL}$ foram classificados como portadores de dislipidemia ${ }^{4}$. Quanto à HAS foi realizada a aferição da pressão arterial, segundo procedimentos padronizados ${ }^{18}$, tendo sido efetuadas duas medidas em momentos distintos. Considerou-se como casos de HAS os adultos que apresentaram pressão arterial sistóli$\mathrm{ca} \geq 140 \mathrm{mmHg}$ e/ou de pressão arterial diastólica $\geq 90 \mathrm{mmHg}$ ou que relataram o uso de medicação anti-hipertensiva.

As variáveis independentes foram agrupadas em:

a) demográficas: sexo (masculino e feminino) e faixa etária (20-29, 30-39, 40-49 e 50-59);

b) socioeconômicas: classe econômica (B1/ B2, C1/C2 e D/E), a qual foi avaliada com base no critério da Associação Brasileira de Empresas de Pesquisa - $\mathrm{ABEP}^{19}$ e definida a partir de um sistema de pontos que considera a posse de bens e o grau de instrução do chefe de família; escolaridade (0-8, 9-11 e $\geq 12$ anos de estudo); raça autorreferida (branca, preta, parda e outras); ocupação (trabalho esporádico, empregado/autônomo, não trabalha/desempregado e benefícios sociais) e saneamento básico - destino do lixo, destino dos dejetos e saneamento básico (rede geral/coleta pública e outros); c) comportamentais: consumo alimentar semanal - feijão, frutas, verduras e legumes, doces e refrigerantes ou sucos artificiais (inferior a cinco vezes e igual ou maior a cinco vezes na semana); consumo de sal (adequado e alto); carnes com excesso de gordura (não e sim). Estas variáveis, referentes ao consumo alimentar, incluindo o consumo de sal, foram investigadas e classificadas com base no questionário de consumo semanal utilizado pelo Ministério da Saúde ${ }^{20}$. A atividade física foi classificada em "suficientemente ativa" e "insuficientemente ativa", teve como referência o "Questionário Internacional de Atividade Física - IPAQ", em sua versão curta ${ }^{21}$, que contém quatro categorias do nível de atividade física: muito ativo, ativo, irregularmente ativo e sedentário. Para fins de análise, a variável foi recategorizada em: "suficientemente ativa" (muito ativa + ativa), aplicada às pessoas que referiram praticar atividade vigorosa com frequência maior ou igual a três vezes na semana por 20 minutos ou mais ou que praticavam atividade moderada ou de caminhada com frequência maior ou igual a cinco vezes na semana, no mínimo 30 minutos ou, ainda, qualquer atividade que somada fosse equivalente a uma frequência maior ou igual a cinco vezes na semana e maior ou igual a 150 minutos na semana (caminhada + atividade moderada + atividade vigorosa) e "insuficientemente ativa" (irregularmente ativa + sedentário), as pessoas não enquadradas no critério supracitado. O tabagismo foi classificado em: fumante atual, ex-fumante e não fuma. Para avaliar o consumo abusivo ou não de bebida alcoólica (sim e não), foi considerada a ingestão de cinco ou mais doses (homens) e quatro ou mais doses (mulheres), em uma única ocasião, pelo menos uma vez nos últimos 30 dias'. Foi considerada uma dose de bebida alcoólica a quantidade correspondente a meia garrafa ou uma lata de cerveja, uma taça de vinho ou uma dose de cachaça, whisky ou qualquer outra bebida alcoólica destilada ${ }^{20}$

d) características relativas à saúde: Índice de Massa Corporal (IMC) - peso adequado: IMC $<25 \mathrm{Kg} / \mathrm{m}^{2}$ e excesso de peso: $\mathrm{IMC} \geq 25 \mathrm{Kg} / \mathrm{m}^{2}$. O peso e estatura foram aferidos e os adultos foram classificados de acordo com as recomendações da Organização Mundial da Saúde ${ }^{22}$. A aferição do peso deu-se em balança digital, da marca Seca 876, com capacidade de até $250 \mathrm{Kg}$ e escala de 100 gramas, com os indivíduos descalços, usando indumentária mínima e sem qualquer objeto nos bolsos, nas mãos ou na cabeça. Para medição da altura, utilizou-se estadiômetro portátil, marca Alturaexata, Ltda., milimetrado, com precisão de até $1 \mathrm{~mm}$ em toda a sua extensão. Os voluntários 
ficaram em posição ereta, descalços, com membros superiores pendentes ao longo do corpo. A percepção da própria saúde (autorreferida) foi classificada em: muito bom/bom, regular e ruim; a categoria "muito ruim" não foi considerada no questionário da pesquisa primária.

Inicialmente, realizaram-se análises descritivas para caracterizar a distribuição de frequência das variáveis. Para análise de possíveis colinearidades entre as variáveis independentes foi usado o teste de correlação de Pearson, considerando valores de $r \geq 0,50$. Posteriormente, foram efetuadas análises bivariadas, através da Regressão de Poisson simples, para evidenciar possíveis associações de DCNT com as variáveis independentes. A categoria das variáveis que apresentou a melhor condição ou a menor prevalência do desfecho foi considerada como referência para as análises. As variáveis que apresentaram nas análises bivariadas valor de $p<0,20$ foram selecionadas para análise de regressão multivariada, de forma hierarquizada. Seguindo uma modelagem por blocos, as variáveis socioeconômicas (escolaridade, ocupação e destino de lixo) foram incluídas no primeiro bloco, mantendo-se no modelo as variáveis que evidenciaram associação estatisticamente significante com a DCNT $(p<0,05)$. Esse procedimento foi repetido para os blocos seguintes, sendo assim constituídos: segundo bloco (variáveis comportamentais - consumo de doce e atividade física), terceiro bloco (variáveis referente às características relativas à saúde - IMC e percepção da própria saúde) e quarto bloco (variáveis demográficas - sexo e faixa etária). As análises foram realizadas por meio de Regressão de Poisson, com ajuste robusto do erro padrão. Os resultados foram expressos por RP ajustadas com respectivos Intervalos de Confiança de 95\% (IC95\%). As análises estatísticas foram processadas com o auxílio de software SPSS, versão 13.0 (SPSS Inc., Chicago, Estados Unidos) e Stata, versão 14.0 (Stata Corp.,College Station, Estados Unidos).

A pesquisa primária foi aprovada pelo Comitê de Ética de Pesquisa do Instituto de Medicina Integral Professor Fernando Figueira (CEP - IMIP), de acordo com os preceitos da Resolução do CNS $466 / 12^{23}$. Os adultos investigados foram informados quanto à participação voluntária na Pesquisa e assinaram o Termo de Consentimento Livre e Esclarecido (TCLE).

\section{Resultados}

A prevalência de DCNT (diabetes ou hipertensão ou dislipidemia) encontrada entre os adultos re- sidentes na comunidade estudada foi de $56,7 \%$.

Ao se analisar as doenças segundo variáveis demográficas e socioeconômicas (Tabela 1), verificou-se que houve uma predominância entre os homens $(60,8 \%)$, quando comparado às mulheres $(54,6 \%)$. Foi observado um aumento progressivo das prevalências de DCNT a partir da faixa de 30-39 anos, com maior frequência na faixa de 50-59 anos (80,5\%). Em relação às classes econômicas, foi observada uma maior prevalência das DCNT entre as classes D/E (57,7\%), ressaltandose, entretanto, que as demais classes apresentaram valores semelhantes. No que se refere à escolaridade, maiores frequências das doenças entre aqueles com menores níveis de instrução foram encontradas com valores de $62 \%(0-8$ anos de estudo) e 60,5\% (9-11 anos de estudo). Evidenciou-se uma maior prevalência de DCNT entre os adultos da raça parda e outras $(57,4 \%)$ e para a ocupação profissional foi observada na categoria "benefícios sociais" (73,3\%). Para as variáveis relativas ao saneamento básico (destino de lixo, destino de dejetos e abastecimento de água) foram encontrados valores em torno de $57 \%$ entre aqueles que possuíam coleta pública/rede geral. Na Tabela 2 verificou-se que variáveis relativas ao consumo alimentar e de sal apresentaram prevalências de DCNT semelhantes, oscilando entre $50,9 \%$ (consumo de doces $\geq 5$ vezes na semana) e $58,8 \%$ (consumo de frutas $\geq 5$ vezes na semana). Quanto à prática de atividade física constatouse maior prevalência na categoria "insuficientemente ativa" $(63,2 \%)$ e em relação ao tabagismo foi maior entre aqueles que eram ex-fumantes (68\%). No que se refere ao consumo abusivo de bebida alcoólica, os resultados foram similares, com prevalência de $57 \%$. Observou-se uma maior prevalência de DCNT entre os indivíduos com excesso de peso, IMC $\geq 25 \mathrm{~kg} / \mathrm{m}^{2}(64,3 \%)$. Para a percepção da própria saúde, a maior prevalência foi encontrada entre os que referiram estado de saúde ruim $(76,4 \%)$. Após ajuste dos fatores associados mediante análise multivariada hierarquizada (Tabela 3), permaneceram quatro variáveis: escolaridade (bloco I), IMC e percepção da própria saúde (bloco III), sexo e faixa etária (bloco IV), as quais se associaram estatisticamente ao desfecho em estudo $(p<0,05)$.

\section{Discussão}

As populações residentes em áreas urbanas carentes (favelas tradicionais ou "aglomerados urbanos subnormais") representam cerca de 11 milhões de habitantes no Brasil (6\%), ou seja, quase o dobro 
Tabela 1. Prevalência e razão de prevalência bruta de Doenças Crônicas não Transmissíveis de adultos (20-59 anos), segundo variáveis demográficas e socioeconômicas. Comunidade dos Coelhos. Recife, 2014.

\begin{tabular}{|c|c|c|c|c|c|c|}
\hline \multirow{2}{*}{ Variáveis } & \multicolumn{2}{|c|}{ Amostra } & \multicolumn{2}{|c|}{ DCNT $^{\mathrm{a}}$} & \multirow{2}{*}{$\begin{array}{l}\text { RP bruta } \\
\text { (IC 95\%) }\end{array}$} & \multirow{2}{*}{ p } \\
\hline & $\mathbf{n}$ & $\%$ & $\mathbf{n}$ & $\%$ & & \\
\hline \multicolumn{7}{|l|}{ Demográficas } \\
\hline Sexo & 631 & & & & & 0,125 \\
\hline Feminino & 414 & 65,6 & 226 & 54,6 & 1,0 & \\
\hline Masculino & 217 & 34,4 & 132 & 60,8 & $1,11(0,97-1,28)$ & \\
\hline Faixa etária (anos) & 631 & & & & & $<0,001$ \\
\hline $20-29$ & 125 & 19,8 & 34 & 27,2 & 1,0 & \\
\hline $30-39$ & 174 & 27,6 & 85 & 48,9 & $1,79(1,29-2,48)$ & \\
\hline $40-49$ & 168 & 26,6 & 106 & 63,7 & $2,34(1,71-3,20)$ & \\
\hline $50-59$ & 164 & 26,0 & 129 & 80,5 & $2,95(2,20-3,98)$ & \\
\hline \multicolumn{7}{|l|}{ Socioeconômicas } \\
\hline Classe econômica & 631 & & & & & 0,940 \\
\hline $\mathrm{B} 1 / \mathrm{B} 2\left(31 / 2-7 \mathrm{SM}^{\mathrm{b}}\right)$ & 37 & 5,9 & 21 & 56,8 & 1,0 & \\
\hline $\mathrm{C} 1 / \mathrm{C} 2\left(1 \frac{1}{2}-2 \mathrm{SM}\right)$ & 372 & 58,9 & 209 & 56,2 & $0,99(0,74-1,33)$ & \\
\hline $\mathrm{D} / \mathrm{E}(1 \mathrm{SM})$ & 222 & 35,2 & 128 & 57,7 & $1,01(0,75-1,37)$ & \\
\hline Escolaridade (anos) & $620^{j}$ & & & & & 0,008 \\
\hline$\geq 12$ & 225 & 36,3 & 108 & 48,0 & 1,0 & \\
\hline $9-11$ & 129 & 20,8 & 78 & 60,5 & $1,26(1,04-1,53)$ & \\
\hline $0-8$ & 266 & 42,9 & 165 & 62,0 & $1,29(1,09-1,52)$ & \\
\hline Raça/etnia & $628^{j}$ & & & & & 0,803 \\
\hline Preta & 75 & 11,9 & 40 & 53,3 & 1,0 & \\
\hline Branca & 116 & 18,5 & 65 & 56,0 & $1,05(0,80-1,37)$ & \\
\hline Parda e outras ${ }^{c}$ & 437 & 69,6 & 251 & 57,4 & $1,08(0,86-1,35)$ & \\
\hline Ocupação & $630^{j}$ & & & & & 0,038 \\
\hline Trabalho esporádico $^{d}$ & 112 & 17,7 & 56 & 50,0 & 1,0 & \\
\hline Empregado/Autônomo & 296 & 47,0 & 163 & 55,1 & $1,10(0,89-1,36)$ & \\
\hline Não trabalha/Desempregado ${ }^{e}$ & 192 & 30,5 & 116 & 60,4 & $1,21(0,97-1,50)$ & \\
\hline Benefícios Sociais $^{\mathrm{f}}$ & 30 & 4,8 & 22 & 73,3 & $1,47(1,10-1,95)$ & \\
\hline \multicolumn{7}{|l|}{ Saneamento Básico } \\
\hline Destino do lixo & 631 & & & & & 0,193 \\
\hline Coleta pública & 567 & 89,8 & 327 & 57,7 & 1,0 & \\
\hline Outros $^{g}$ & 64 & 10,2 & 31 & 48,4 & $0,84(0,64-1,09)$ & \\
\hline Destino dos dejetos & 631 & & & & & 0,895 \\
\hline Rede geral & 307 & 48,6 & 175 & 57,0 & 1,0 & \\
\hline Outros $^{\mathrm{h}}$ & 324 & 51,4 & 183 & 56,3 & $0,99(0,86-1,13)$ & \\
\hline Abastecimento de água & 631 & & & & & 0,294 \\
\hline Rede geral & 592 & 93,8 & 340 & 57,4 & 1,0 & \\
\hline Outros $^{\mathrm{i}}$ & 39 & 6,2 & 18 & 46,2 & $0,80(0,57-1,14)$ & \\
\hline $\begin{array}{l}\text { RP: Razão de prevalência IC95\%: Interv } \\
\text { - HAS, DM ou dislipidemia. b: SM- salá } \\
\text { ambulante. e:nunca trabalhou e donas d } \\
\text { pensionista, benefícios provisórios. g: qu } \\
\text { com e sem tampa, curso de água, céu ab } \\
\text { valores amostrais em algumas variáveis } \\
\text { inconsistência de dados. }\end{array}$ & $\begin{array}{l}\text { inça } \\
\text { c: o } \\
\text { trab } \\
\text { reno } \\
\text { is sit } \\
\text { perd }\end{array}$ & $\begin{array}{l}\text { a: DC } \\
\text { aças at } \\
\text { las est } \\
\text { deposi } \\
\text { i: poço }\end{array}$ & $\begin{array}{l}\text { resença } \\
\text { e indís } \\
\text { sempre } \\
\text { m caça } \\
\text { na, cha }\end{array}$ & $\begin{array}{l}\text { lo me } \\
\text { d: trab } \\
\text { há } 30 \mathrm{c} \\
\text { ara co } \\
\text { e dema } \\
\text { uestio }\end{array}$ & $\begin{array}{l}\text { s uma das seguintes } \\
\text { o esporádico/biscate } \\
\text { s ou mais. f: aposent } \\
\text { ta e demais situações } \\
\text { situações. j: As difere } \\
\text { rios incompletos e/o }\end{array}$ & $\begin{array}{l}\text { loenças } \\
\text { ro/ } \\
\text { do, } \\
\text { h: fossa } \\
\text { hças de } \\
\text { por }\end{array}$ \\
\hline
\end{tabular}

da proporção encontrada em $1991(3,1 \%)^{15}$. Há de se considerar a realidade complexa das favelas, que é marcada pela desigualdade, condições precárias de saneamento, habitações insalubres, falta ou insuficiência de serviços de saúde. Não obs- tante, características semelhantes de vulnerabilidade socioeconômica também podem ser identificadas em populações residentes em outras áreas urbanas, considerando-se as diferentes condições de acesso da população a diferentes políticas pú- 
Tabela 2. Prevalência e razão de prevalência bruta de Doenças Crônicas não Transmissíveis de adultos (20-59 anos), segundo variáveis comportamentais e características relativas à saúde. Comunidade dos Coelhos. Recife, 2014.

\begin{tabular}{|c|c|c|c|c|c|c|}
\hline \multirow{2}{*}{ Variáveis } & \multicolumn{2}{|c|}{ Amostra } & \multicolumn{2}{|c|}{ DCNT $^{\mathrm{a}}$} & \multirow{2}{*}{$\begin{array}{c}\text { RP bruta } \\
\text { IC } 95 \%\end{array}$} & \multirow{2}{*}{$\mathbf{p}$} \\
\hline & $\mathbf{n}$ & $\%$ & $\mathbf{n}$ & $\%$ & & \\
\hline \multicolumn{7}{|l|}{ Comportamentais } \\
\hline \multicolumn{7}{|l|}{ Consumo alimentar semanal } \\
\hline Feijão & 631 & & & & & 0,492 \\
\hline$\geq 5$ vezes & 430 & 68,1 & 248 & 57,7 & 1,0 & \\
\hline$<5$ vezes & 201 & 31,9 & 110 & 54,7 & $0,95(0,81-1,10)$ & \\
\hline Verduras e legumes & 631 & & & & & 0,392 \\
\hline$\geq 5$ vezes & 271 & 43,0 & 159 & 58,7 & 1,0 & \\
\hline$<5$ vezes & 360 & 57,0 & 199 & 55,3 & $0,94(0,82-1,08)$ & \\
\hline Frutas & 631 & & & & & 0,488 \\
\hline$\geq 5$ vezes & 194 & 30,7 & 114 & 58,8 & 1,0 & \\
\hline$<5$ vezes & 437 & 69,3 & 244 & 55,8 & $0,95(0,82-1,10)$ & \\
\hline Refrigerante ou suco artificial & 631 & & & & & 0,283 \\
\hline$<5$ vezes & 433 & 68,6 & 252 & 58,2 & 1,0 & \\
\hline$\geq 5$ vezes & 198 & 49,4 & 106 & 53,5 & $0,91(0,79-1,07)$ & \\
\hline Doces & 631 & & & & & 0,180 \\
\hline$<5$ vezes & 515 & 81,6 & 299 & 58,1 & 1,0 & \\
\hline$\geq 5$ vezes & 116 & 31,4 & 59 & 50,9 & $0,87(0,72-1,06)$ & \\
\hline Carnes com excesso de gordura & 631 & & & & & 0,490 \\
\hline Não & 430 & 68,1 & 240 & 55,8 & 1,0 & \\
\hline Sim & 201 & 31,9 & 118 & 58,7 & $1,05(0,91-1,21)$ & \\
\hline Consumo de sal & 631 & & & & & 0,964 \\
\hline Adequado & 539 & 85,4 & 306 & 56,8 & 1,0 & \\
\hline Alto & 92 & 14,6 & 52 & 56,5 & $0,99(0,82-1,21)$ & \\
\hline Atividade física & 631 & & & & & 0,068 \\
\hline Suficientemente ativo & 495 & 78,4 & 272 & 54,9 & 1,0 & \\
\hline Insuficientemente ativo & 136 & 21,6 & 86 & 63,2 & $1,15(0,99-1,34)$ & \\
\hline Consumo abusivo de bebida alcoólica & 631 & & & & & 0,992 \\
\hline Não & 400 & 63,4 & 227 & 56,8 & 1,0 & \\
\hline Sim & 231 & 36,6 & 131 & 56,7 & $1,00(0,87-1,14)$ & \\
\hline Tabagismo & 631 & & & & & 0,407 \\
\hline Não fumante & 468 & 74,2 & 264 & 56,4 & 1,0 & \\
\hline Fumante & 138 & 21,9 & 77 & 55,8 & $0,99(0,83-1,17)$ & \\
\hline Ex-fumante & 25 & 3,9 & 17 & 68,0 & $1,20(0,91-1,60)$ & \\
\hline \multicolumn{7}{|l|}{ Características relativas à saúde } \\
\hline $\operatorname{IMC}\left(\mathrm{kg} / \mathrm{m}^{2}\right)^{\mathrm{b}}$ & 631 & & & & & $<0,001$ \\
\hline$<25$ & 186 & 29,5 & 72 & 38,7 & 1,0 & \\
\hline$\geq 25$ & 445 & 70,5 & 286 & 64,3 & $1,66(1,37-2,01)$ & \\
\hline Percepção da própria saúde & 631 & & & & & $<0,001$ \\
\hline Muito bom/Bom & 37 & 5,9 & 15 & 40,5 & 1,0 & \\
\hline Regular & 222 & 35,2 & 147 & 66,2 & $1,39(1,20-1,60)$ & \\
\hline Ruim & 55 & 8,7 & 42 & 76,4 & $1,60(1,33-1,92)$ & \\
\hline
\end{tabular}

blicas, como por exemplo, o acesso ao saneamento básico.

No presente estudo, a elevada prevalência de DCNT (56,7\%) identificada foi semelhante a que foi verificada em uma comunidade de baixa renda dos Estados Unidos (60,2\%) por Smolen et al. ${ }^{24}$. Em estudo representativo no Brasil, identificouse uma magnitude de $75,3 \%$ de DCNT na região nordeste, a qual foi identificada como uma das regiões com as maiores taxas de doenças crônicas. 
Tabela 3. Modelo final. análise multivariada hierarquizada de Doenças Crônicas não Transmissíveis de adultos(20-59 anos) Comunidade dos Coelhos. Recife, 2014.

\begin{tabular}{|c|c|c|c|c|}
\hline Bloco $^{a}$ & Variáveis & RP ajustada & IC $95 \%$ & $\mathbf{p}$ \\
\hline \multirow[t]{4}{*}{ I } & Escolaridade (anos) & & & 0,021 \\
\hline & $\geq 12$ & 1,0 & 1,0 & \\
\hline & $9-11$ & 1,24 & $1,05-1,48$ & \\
\hline & $0-8$ & 1,06 & $0,90-1,24$ & \\
\hline \multirow[t]{7}{*}{ III } & $\operatorname{IMC}\left(\mathrm{kg} / \mathrm{m}^{2}\right)^{\mathrm{b}}$ & & & $<0,001$ \\
\hline & $<25$ & 1,0 & 1,0 & \\
\hline & $\geq 25$ & 1,52 & $1,26-1,82$ & \\
\hline & Percepção da própria saúde & & & 0,021 \\
\hline & Muito bom/Bom & 1,0 & 1,0 & \\
\hline & Regular & 1,20 & $1,04-1,38$ & \\
\hline & Ruim & 1,22 & $1,02-1,47$ & \\
\hline \multirow[t]{8}{*}{ IV } & Sexo & & & 0,030 \\
\hline & Feminino & 1,0 & 1,0 & \\
\hline & Masculino & 1,15 & $1,01-1,31$ & \\
\hline & Faixa etária (anos) & & & $<0,001$ \\
\hline & $20-29$ & 1,0 & 1,0 & \\
\hline & $30-39$ & 1,59 & $1,15-2,19$ & \\
\hline & $40-49$ & 2,07 & $1,53-2,82$ & \\
\hline & $50-59$ & 2,62 & $1,94-3,54$ & \\
\hline
\end{tabular}

RP: Razão de prevalência IC95\%: Intervalo de Confiança = 95\%. a: Bloco I ajustado por ocupação e destino de lixo. Bloco II foi composto pelas variáveis consumo de doce e atividade física, porém não permaneceram no modelo porque não foram significativas. Bloco III ajustado por escolaridade. Bloco IV ajustado por escolaridade, IMC e percepção da própria saúde. b: IMC: Índice de Massa Corporal.

A carga elevada deste grupo de doenças nesta região pode ser reflexo de piores condições de vida e de acesso aos serviços de saúde para controle dos fatores de risco e para controle das doenças crônicas, determinando mortes mais precoces e maior incidência de sequelas ${ }^{11}$.

Em relação à escolaridade, encontrou-se maior prevalência de DCNT entre aqueles com menos de oito anos de estudo. Achados semelhantes foram identificados em pesquisas nacionais e internacionais ${ }^{25-28}$. De acordo com Braveman et al. ${ }^{29}$, as pessoas com menos escolaridade e que vivem em localizações geográficas menos favorecidas, são desproporcionalmente afetadas por doenças crônicas, muitas vezes como resultado de desvantagens sociais e maior vulnerabilidade.

A prevalência de DCNT foi maior no sexo masculino do que no feminino, com diferença estatisticamente significante. Rocha-Brischiliari et al. ${ }^{30} \mathrm{em}$ estudo em Maringá-PR, também encontraram predominância de doenças crônicas entre os homens, porém não foi constatada associação. Já Barreto e Figueiredo ${ }^{25} \mathrm{e}$ Almeida et al ${ }^{26}$ em estudos no país demostraram associação entre sexo e DCNT, contudo, a maior prevalência foi entre as mulheres. Tais divergências podem apontar que a diferença entre os sexos para a prevalência de DCNT ainda não está bem estabelecida na litera- tura científica, principalmente em pesquisas com dados aferidos, uma vez que os estudos citados acima utilizaram dados autorreferidos de doença.

Observou-se associação estatisticamente significante entre as DCNT e a faixa etária, com aumento linear da prevalência do problema, corroborando com Bhojani et al. ${ }^{31}$ em estudo com adultos pobres da Índia e no Brasil por Rocha -Brischiliari et al. ${ }^{30}$.

$\mathrm{O}$ problema predominou entre os adultos com IMC $\geq 25$, representado pelo excesso de peso, com diferença estatisticamente significante. Gigante et al. ${ }^{32}$ ao realizarem um estudo com base nos dados obtidos pelo Sistema de Vigilância de Fatores de Risco e Proteção para Doenças Crônicas por Inquérito Telefônico (Vigitel), demonstraram que os indivíduos com IMC mais elevado tiveram maior prevalência de doenças crônicas autorreferidas. Estudos tem evidenciado uma tendência de aumento do excesso de peso em populações menos favorecidas no mundo ${ }^{29,33-35}$, caracterizando uma situação bastante preocupante, uma vez que o excesso de peso está intimamente relacionado com o risco das Doenças Crônicas Não Transmissíveis.

Verificou-se que os adultos que referiram "estado de saúde "regular" e "ruim" apresentaram uma maior risco para DCNT em compara- 
ção com a categoria "muito bom/bom" (respectivamente RP: 1,20; IC95\%: 1,04-1,38; RP: 1,22; IC95\%: 1,02-1,47). No Brasil, Theme Filha et al. ${ }^{3}$ também encontraram essa condição, assim como Brow et al. ${ }^{28}$ nos Estados Unidos (Los Angeles). Segundo Idler e Benyamini ${ }^{36}$, indivíduos que auto-avaliam sua saúde como ruim tendem a apresentar mais comportamentos de risco para saúde.

Uma alimentação inadequada, rica em gorduras, pobre em frutas, legumes e verduras, está associada ao aparecimento de diversas doenças crônicas como hipercolesterolemia, hipertensão arterial, diabetes mellitus e câncer ${ }^{8} \mathrm{e}$ a realidade atual aponta para um consumo inadequado de alimentos, sobretudo, em populações de baixa renda $a^{37,38}$. Entretanto, não foi encontrada associação entre ter pelo menos uma DCNT e as variáveis de consumo alimentar. A prevalência de doenças crônicas foi praticamente a mesma entre as categorias de alimentos classificados como de risco ou de proteção. Isso pode ser atribuído ao possível viés de falsidade intencional, que ocorre quando os indivíduos tendem a relatar hábitos saudáveis quando na realidade não o fazem. Essa tendência é frequentemente observada em estudos populacionais sobre consumo alimentar ${ }^{39,40}$. Ainda deve ser considerado que a possibilidade do viés de causalidade reversa, ou seja, as mudanças no modo de vida podem decorrer da presença da doença. E nesse caso, é um indicador positivo, pois sinaliza cuidado com a saúde entre aqueles que estão doentes.

Também não foi constatada associação entre atividade física e DCNT. Contudo, a inatividade física é um importante fator de risco para essas doenças ${ }^{8}$. Considerando-se os baixos níveis da prática de atividade física verificados no país nos últimos anos ${ }^{41}$ e que também foi confirmada nesta investigação (21,6\% dos adultos eram insuficientemente ativos), um pequeno aumento do nível de atividade física na população brasileira representaria uma intervenção importante para a melhoria da saúde coletiva, dado seu reconhecido papel na prevenção primária de hipertensão arterial, diabetes tipo 2, doença coronariana, acidente vascular cerebral, osteoporose, alguns tipos de câncer, dislipidemia, obesidade e depressão ${ }^{42}$.

Considerando que o consumo abusivo de álcool e o tabagismo são potenciais fatores de risco para as doenças crônicas ${ }^{4,30,43}$, no presente estudo essa associação não foi observada. Contudo, este resultado pode ter sido comprometido pelo tamanho amostral, bem como pelo fato da coleta dessas variáveis não terem sido bem discriminadas, por se tratar de limitações do questionário, podendo não refletir a condição real do comportamento dos indivíduos.

O substancial crescimento desses aglomerados não tem sido devidamente analisado e acompanhado, tendo em vista a escassez de estudos científicos, que permanece rudimentar quando comparados com os muitos estudos em áreas não faveladas $^{44}$. Ressalta-se a necessidade de se intensificar os estudos relacionados com a DCNT, que se apresentam com elevadas prevalências no atual perfil epidemiológico internacional e nacional e em diferentes contextos socioeconômicos, inclusive os mais carentes.

\section{Conclusões}

A elevada prevalência de DCNT e os fatores associados, identificados neste estudo, são de fundamental importância para auxiliar na elaboração e implementação de estratégias de controle, prevenção e promoção de saúde, necessárias para diminuir o alarmante índice de morbidade crônica nessa e em outras áreas menos favorecidas. Os fatores associados significantemente à DCNT que compuseram o modelo multivariado hierarquizado foram: escolaridade, IMC, percepção de saúde, idade e faixa etária.

Considerando a escassez de estudos em áreas urbanas pobres e as relevantes prevalências das DCNT encontradas, o presente trabalho aponta a importância do monitoramento dessas doenças e de seus fatores associados. Um maior e mais detalhado conhecimento do quadro epidemiológico desse problema possibilitará um melhor planejamento e controle dessas doenças e a priorização de ações de promoção à saúde da população nessas áreas.

\section{Colaboradores}

SPSC Melo, EAP Cesse, PIC Lira, A Rissin e M Batista Filho contribuíram na concepção, delineamento do estudo, análise e interpretação dos resultados, redação e revisão crítica do conteúdo do estudo. RSBLC Cruz participou na concepção, delineamento e redação do estudo. Todos os autores aprovaram a versão final do manuscrito e declararam serem responsáveis por todos os aspectos do trabalho, garantindo sua precisão e integridade. 


\section{Referências}

1. Mendes EV. O cuidado das condições crônicas na atenção primária à saúde: o imperativo da consolidação da estratégia da saúde da família. Brasília: Organização Pan-Americana da Saúde; 2012.

2. Goulart FAA. Doenças crônicas não transmissiviveis: estratégias de controle e desafios para os sistemas de saúde. Brasília: Organização Pan-Americana da Saúde; 2011.

3. Theme Filha MM, Souza Júnior PRB, Damacena GN, Szwarcwald CL. Prevalência de doenças crônicas não transmissíveis e associação com autoavaliação de saúde: Pesquisa Nacional de Saúde, 2013. Rev. Bras. Epidemiol 2015; 18(Supl. 2):83-96.

4. Xavier HT, Izar MC, Faria Neto JR, Assad MH, Rocha VZ, Sposito AC, Fonseca FA, Santos JE, Santos RD, Bertolami MC, Faludi AA, Martinez TLR, Diament J, Guimarães A, Forti NA, Moriguchi E, Chagas ACP, Coelho OR, Ramires JAF. V Diretriz Brasileira de Dislipidemias e Prevenção da Aterosclerose. Arq Bras. Cardiol. 2013; 101(4 Supl. 1):1-20.

5. Bloom DE, Cafiero ET, Jané-Llopis E, AbrahamsGessel S, Bloom LR, Fathima S, Feigl AB, Gaziano T, Mowafi M, Pandya A, Prettner K, Rosenberg L, Seligman B, Stein AZ, Weinstein C. The global economic burden of non-communicable diseases: report by the World Economic Forum and the Harvard School of Public Health [Internet]. Geneva: World Economic Forum; 2011. [cited 2016 Mar 12]. Available from: http:// www3.weforum.org/docs/WEF_Harvard_HEa lEconomicBurdenNonCommunicableDiseases_ 2011.pdf

6. Bonita R, Magnusso R, Bovet P, Zhao D, Mata DC, McKee M, Hospedales J, de Courten M, Capewell S, Beaglehole R; Lancet NCD Action Group. Country actions to meet UN commitments on non-communicable diseases: a stepwise approach. Lancet 2013; 381(9866):575-584.

7. Abegunde DO, Mathers CD, Adam T, Ortegon M, Strong K. The burden and costs of chronic diseases in low-income and middle-income countries. Lancet 2007; 370(9603):1929-1938.

8. Brasil. Ministério da Saúde (MS). Plano de ações estratégicas para o enfrentamento das doenças crônicas não transmissíveis (DCNT) no Brasil 2011-2022 [Internet]. Brasília: MS; 2011. [acessado 2016 Mar 11]. Disponível em: http://bvsms.saude.gov.br/bvs/publicacoes/plano_acoes_enfrent_dent_2011.pdf

9. Alwan A, Maclean DR, Riley LM, d'Espaignet ET, Mathers CD, Stevens GA, Bettcher D. Monitoring and surveillance of chronic non-communicable diseases: progress and capacity in high-burden countries. Lancet 2010; 376(9755):1861-1868.

10. Malta DC, Moura L, Prado RR, Escalant JC, Schmidt MI, Duncan BB. Mortalidade por doenças crônicas não transmissíveis no Brasil e suas regiões, 2000 a 2011. Epidemiol Serv Saúde 2014, 23(4):599-608.

11. Leite CI, Valente GJ, Schramm JMA, Daumas RP, Rodrigues RN, Santos MF, Oliveira AF, Silva RS, Campos MR, Mota JC. Carga de doença no Brasil e suas regiões, 2008. Cad. Saúde Pública 2015; 31(7):15511564.

12. Casado L, Vianna LM, Thuler LCS. Fatores de risco para Doenças Crônicas Não Transmissíveis no Brasil: uma revisão sistemática. Rev. Bras. Cancerol 2009; 55(4):379-388.
13. World Health Organization (WHO). Global status report on noncommunicable diseases 2010. Geneva: WHO; 2011.

14. World Health Organization (WHO). Global status report on noncommunicable diseases 2014 [Internet]. Geneva: WHO; 2014. [acessado 2017 Mar 12]. Disponível em: http://www.who.int/nmh/publications/ ncd-status-report-2014/en/

15. Instituto Brasileiro de Geografia e Estatística (IBGE). Aglomerados subnormais. Censo Demográfico. Rio de Janeiro: IBGE; 2010.

16. Ezeh A, Oyebode O, Satterthwaite D, Chen YF, Ndugwa $\mathrm{R}$, Sartori J, The history, geography, and sociology of slums and the health problems of people who live in slums. Lancet 2016; 389(10068):547-558.

17. American Diabetes Association. Standards of Medical Care in Diabetes. Diabetes Care 2013; 36(Supl. 1):1166.

18. Malachias MVB, Souza WKSB, Plavnik FL, Rodrigues CIS, Brandão AA, Neves MFT, Bortolotto LA, Franco RJS, Poli-de-Figueiredo CE, Jardim PCBV, Amodeo C, Barbosa ECD, Koch V, Gomes MAM, Paula RB, Póvoa RMS, Colombo FC, Ferreira Filho S, Miranda RD, Machado CA, Nobre F, Nogueira AR, Mion Júnior D, Kaiser S, Forjaz CLM, Almeida FA, Martim JFV, Sass N, Drager LF, Muxfeldt E, Bodanese LC, Feitosa AD, Malta D, Fuchs S, Magalhães ME, Oigman W, Moreira Filho O, Pierin AMG, Feitosa GS, Bortolotto MRFL, Magalhães LBNC, Silva ACS, Ribeiro JM, Borelli FAO, Gus M, Passarelli Júnior O, Toledo JY, Salles GF, Martins LC, Jardim TSV, Guimarães ICB, Antonello IC, Lima Júnior E, Matsudo V, Silva GV, Costa LS, Alessi A, Scala LCN, Coelho EB, Souza D, Lopes HF, Gowdak MMG, Cordeiro Júnior AC, Torloni MR, Klein MRST, Nogueira PK, Lotaif LAD, Rosito GBA, Moreno Júnior H. 7o Diretriz Brasileiras de Hipertensão. Arq Bras Cardiol 2016; 107(Supl. 3):1-83.

19. Associação Brasileira de Empresas de Pesquisa (ABEP). Critério de classificação econômica Brasil. São Paulo: ABEP; 2014.

20. Brasil. Ministério da Saúde (MS). Vigitel Brasil 2013: Vigilância de Fatores de Risco e Proteção para Doenças Crônicas por Inquérito Telefônico. Brasília: MS; 2014.

21. Matsudo S, Araújo T, Matsudo V, Andrade D, Andrade E, Oliveira LC, Braggion G. Questionário Internacional de Atividade Física (IPAQ): Estudo de validade e reprodutibilidade no Brasil. Atividade Física e Saúde 2001; 6(2):5-18.

22. World Health Organization (WHO). Physical status: the use and interpretation of anthropometry. Geneva: WHO; 1995.

23. Brasil. Ministério da Saúde (MS). Conselho Nacional de Saúde. Resolução no 466, de 12 de dezembro de 2012. Diário Oficial da União 2013; $13 \mathrm{dez}$.

24. Smolen JR, Roland J, Thorpe JR, Bowie JV, Gaskin DJ, LaVeist TA. Health Insurance and Chronic Conditions in Low-Income Urban Whites. J Urban Health 2014; 91(4):637-647.

25. Barreto SM, Figueiredo RC. Doença crônica, auto-avaliação de saúde e comportamento de risco: diferença de gênero. Rev. Saúde Pública 2009; 43(Supl. 2):38-47. 
26. Almeida MF, Barata RB, Montero CV, Silva ZP. Prevalência de doenças crônicas auto-referidas e utilização de serviços de saúde, PNAD/1998, Brasil. Cien Saude Colet 2002; 7(4):743-756.

27. Kraja F, Kraja B, Mone I, Harizi I, Babameto A, Burazeri G. Self-reported Prevalence and Risk Factors of Non-communicable Diseases in the Albanian Adult Population. Med Arch 2016; 70(3):208-212.

28. Brown AF, Ang A, Pebley AR. The Relationship Between Neighborhood Characteristics and Self-Rated Health for Adults With Chronic Conditions. Am J Public Health 2007; 97(5):926-932.

29. Braveman PA, Kumanyika S, Fielding J, Laveist T, Borrell LN, Manderscheid R, Troutman A. Health disparities and health equity: the issue is justice. Am J Public Health 2011; 101(Supl. 1):149-155.

30. Rocha-Brischiliari SC, Agnolo CMD, Gravena AAF, Lopes TCR, Carvalho MDB, Pelloso SM. Doenças Crônicas não Transmissíveis e Associação com Fatores de Risco. Rev Bras Cardiol 2014; 27(1):35-42.

31. Bhojani U, Beerenahalli TS, Devadasan R, Munegowda CM, Devadasan N, Crieland B. No longer diseases of the wealthy: prevalence and health-seeking for self-reported chronic conditions among urban poor in Southern India. BMC Health Services Research 2013; 13(306):2-10.

32. Gigante DP, Moura EC, Sardinha LMV. Prevalência de excesso de peso e obesidade e fatores associados, Brasil 2006. Rev. Saúde Pública 2009; 43(Supl. 2):83-89.

33. Batista Filho M, Rissin A. A transição nutricional no Brasil: tendências regionais e temporais. Cad. Saúde Pública 2003; 19 (Supl. 1):S181-191.

34. Monteiro CA, Moura EC, Popkin BM. Socioeconomic status and obesity in adult populations of developing countries: a review. Bull World Health Organ 2004; 82(12):940-946.

35. Silva AP, Silva SH, Haniffa R, Liyanage IK, Jayasinghe KS, Katulanda P, Wijeratne CN, Wijeratne S, Rajapakse LC. A cross sectional survey on social, cultural and economic determinants of obesity in a low middle income setting. Int. J. Equity Health 2015; 14:6.

36. Idler EL, Benyamini Y. Self-rated health and mortality: a review of twenty-seven community studies. $J$ Health Soc. Behav 1997;38(1):21-37.

37. Brasil. Ministério da Saúde (MS). O estado da segurança alimentar e nutricional no brasil: Um retrato multidimensional - Relatório 2014. Brasília: MS; 2014.

38. Malta DC, StopaI SR, Iser BPM, Bernal RTI, Claro RM, Nardi ACF. Fatores de risco e proteção para doenças crônicas por inquérito telefônico nas capitais brasileiras, Vigitel 2014. Rev. Bras. Epidemiol 2015; 18(Supl. 2):238-255.
39. Avelino GF, Previdelli NA, Castro MA, Marchioni DML, Fisberg RM. Sub-relato da ingestão energética e fatores associados em estudo de base populacional. Cad. Saúde Pública 2014; 30(3):663-668.

40. Andrade RG. Excesso de peso em homens e mulheres residentes em área urbana: fatores individuais e contexto socioeconômico. Cad. Saúde Pública 2015; 31(Supl. 1):1-11.

41. Hallal PC, Knuth AG, Reis RS, Rombaldi AJ, Malta DC, Iser BPM, Bernal BTI, Florindo AA. Tendências temporais de atividade física no Brasil (2006-2009). Rev. Bras. Epidemiol 2011; 14 (Supl. 1):53-60.

42. Francisco PMSB, Segri NJ, Barros MBA, Malta DC. Desigualdades sociodemográficas nos fatores de risco e proteção para doenças crônicas não transmissíveis: inquérito telefônico em Campinas, São Paulo. Epidemiol Saúde Serv 2015; 24(1):7-18.

43. Malta DC, Iser BPM, Moura L, Bernal RTI, Nascimento AF. Prevalência de fatores de risco e proteção para. doenças crônicas não transmissíveis em adultos: estudo transversal, Brasil, 2011. Epidemiol. Serv. Saúde 2013; 22(3):423-434.

44. Ezeh A, Oyebode O, Satterthwaite D, Chen YF, Ndugwa $\mathrm{R}$, Sartori J. The history, geography, and sociology of slums and the health problems of people who live in slums. Lancet 2016; 389(10068):547-558.
Artigo apresentado em 29/09/2017

Aprovado em 24/01/2018

Versão final apresentada em 26/01/2018 